
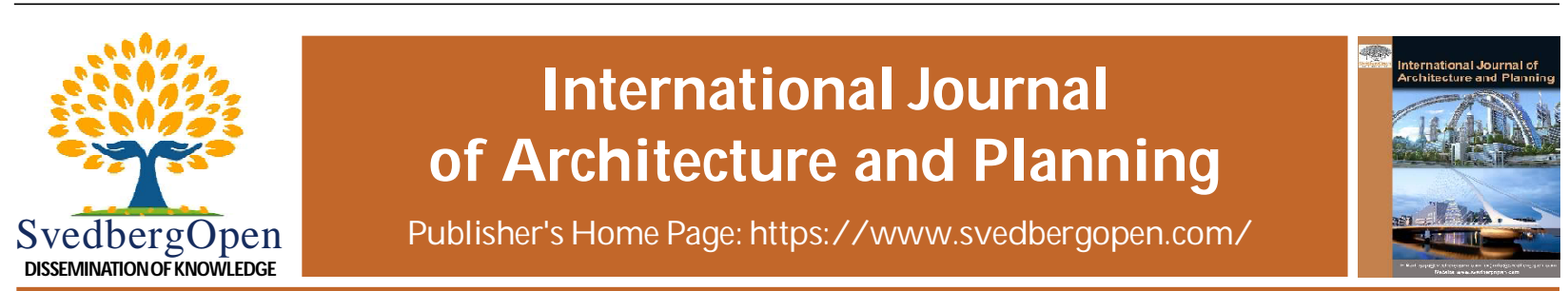

\title{
Study on causes of cracks and its preventive measures in concrete structures of CCEM building, Raipur
}

\author{
Bhumika Das ${ }^{1^{*}}$, Kumar Umnag ${ }^{2}$ and Gopal Murthy ${ }^{3}$ \\ ${ }^{1}$ Associate Professor, Mining Engineering, MATS University, Raipur 492001, Chhattisgarh, India. E-mail: drbhumika@matsuniversity.ac.in \\ ${ }^{2}$ Associate Professor, Mining Engineering, MATS University, Raipur 492001, Chhattisgarh, India. E-mail: umangshrivastava2294@gmail.com \\ ${ }^{3}$ Associate Professor, Mining Engineering, MATS University, Raipur 492001, Chhattisgarh, India. E-mail: bhumikadas777@ gmail.com
}

\section{Article Info}

Volume 1, Issue 1, March 2021

Received : 06 November 2020

Accepted : 10 February 2021

Published : 05 March 2021

doi: 10.51483/IJARP.1.1.2021.30-36

\begin{abstract}
The problem of cracking in building is becoming a difficult puzzle for engineers nowadays. Cracking is an unavoidable response of any structure while designers are trying to eliminate many of the causes of cracking and design tolerance for other factors. We all want our building structurally safe but it is not so easy. Some faulty steps during construction and some unavoidable reasons different type of cracks starts to appear on various structural and non-structural parts of the building. So, timely identification of such cracks and adopting preventive measure are essential. The repair materials and repair techniques are different depending upon forms of cracks according to their positions in structure. Some types of cracks seriously need attention as they are structurally hazardous. In this paper, we will discuss about the problem engineers are facing, i.e., of cracking after construction and what preventive measures should be taken along with the techniques to cure cracks.
\end{abstract}

Keywords: Cracks, Causes of cracking, Preventive measures, Techniques

(C) 2021 International Journal of Architecture and Planning. This is an open access article under the CC BY license (https://creativecommons.org/licenses/by/4.0/), which permits unrestricted use, distribution, and reproduction in any medium, provided you give appropriate credit to the original author(s) and the source, provide a link to the Creative Commons license, and indicate if changes were made.

\section{Introduction}

A crack is a complete or incomplete separation of concrete into two or more parts produced by breaking or fracturing. Concrete structure has been started applying since the mid- $19^{\text {th }}$ century, because of the low quality of cement and at that time the development of concrete structure was slow. Until the end of the $19^{\text {th }}$ century, concrete structure was getting faster development with the development of production, experimental work, computational theory and improvement of construction technique and now it has became one of the most widely used building materials in the modern construction. Cracks are one kind of universal problem of concrete construction as it affects the building artistic and it also destroys the wall's integrity, affects the structure safety even reduce the durability of structure. Cracks develop due to deterioration of concrete or corrosion or reinforcement bars due to poor construction or inappropriate selection of constituent material and by temperature and shrinkage effects.

\subsection{Cracks can be divided into two types}

(A) Structural cracks: These cracks occur due to incorrect design, faulty construction or overloading and these may endanger the safety of a building (CBRI, Roorkee, 2011).

\footnotetext{
* Corresponding author:Bhumika Das, Associate Professor, Mining Engineering, MATS University, Raipur 492001, Chhattisgarh, India. E-mail: drbhumika@matsuniversity.ac.in
} 
(B) Non-structural cracks: They are due to internal forces developed in materials due to moisture variations, temperature variation, crazing, effects of gases, liquids, etc.

\section{Non-structural cracks are:}

- Plastic settlement

- Plastic shrinkage

- Early thermal expansion and contraction

- Long-term drying shrinkage

- Crazing

- Due to corrosion of concrete

- Due to alkali-aggregate reaction

- Sulphate attack

- Due to corrosion of steel

A commonly known classification of cracks on the basis of their width is:

(a) Thin - less than $1 \mathrm{~mm}$ in width

(b) Medium -1 to $2 \mathrm{~mm}$ in width

(c) Wide-more than $2 \mathrm{~mm}$ in width

Cracks may be of uniform width throughout or may be narrow at one end, gradually widening at the other. Cracks may be straight, toothed, stepped, map pattern or random and may be vertical, horizontal or diagonal. Cracks may be only at the surface or may extend to more than one layer of materials.

Principal causes of occurrence of cracks in buildings are as follows:

1. Moisture changes,

2. Thermal variations,

3. Elastic deformation,

4. Creep,

5. Chemical reaction,

6. Foundation movement and settlement of soil, and

7. Vegetation.

\section{Case study}

For a better and thorough understanding a case study was carried out at Central College of Engineering \& Management Raipur, Chhattisgarh. CCEM although being a fairly new institution having started in 2008, most of the structure already have started showing deep cracks at various locations all across the campus which will lead to the decrease in the durability of structure. Most of the structures in the campus including institutional building, hostel, etc. are composite structures where columns are of structural steel slabs being RCC. This combination has led to occurrence of cracks at all the major juncture points of steel and concrete. The use of improper design mix, improper curing along with many other reasons has led to different structural and non-structural cracks. For the cracks all the prominent cracks were identified and classified on its possible causes. Subsequently remedial measures for each of those cracks have been identified and listed. In the month of August 2017, the main building of the college and as well as all the internal rooms was inspected carefully and each type of cracks were photographed and recorded for further observations. The cracks have been categorized on the basis of:

(a) Thin - less than $1 \mathrm{~mm}$ in width,

(b) Medium - 1 to $2 \mathrm{~mm}$ in width, and

(c) Wide-more than $2 \mathrm{~mm}$ in width. 

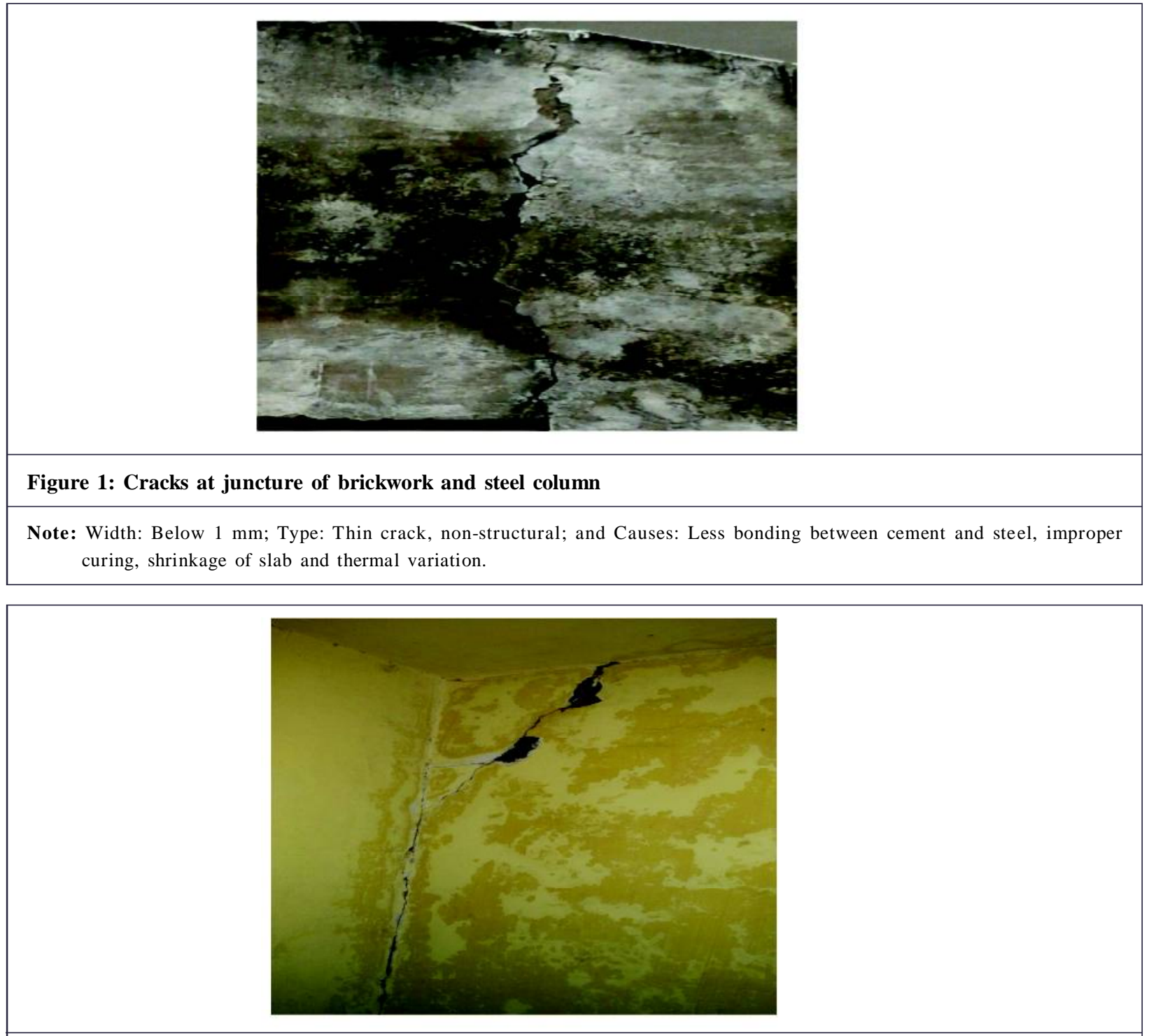

Figure 2: Cracks at corner of sunshade

Note: Width: Above $5 \mathrm{~mm}$, Type: Gap crack; and Causes: Improper cantilevering, due to joint and heavy exposure to water.

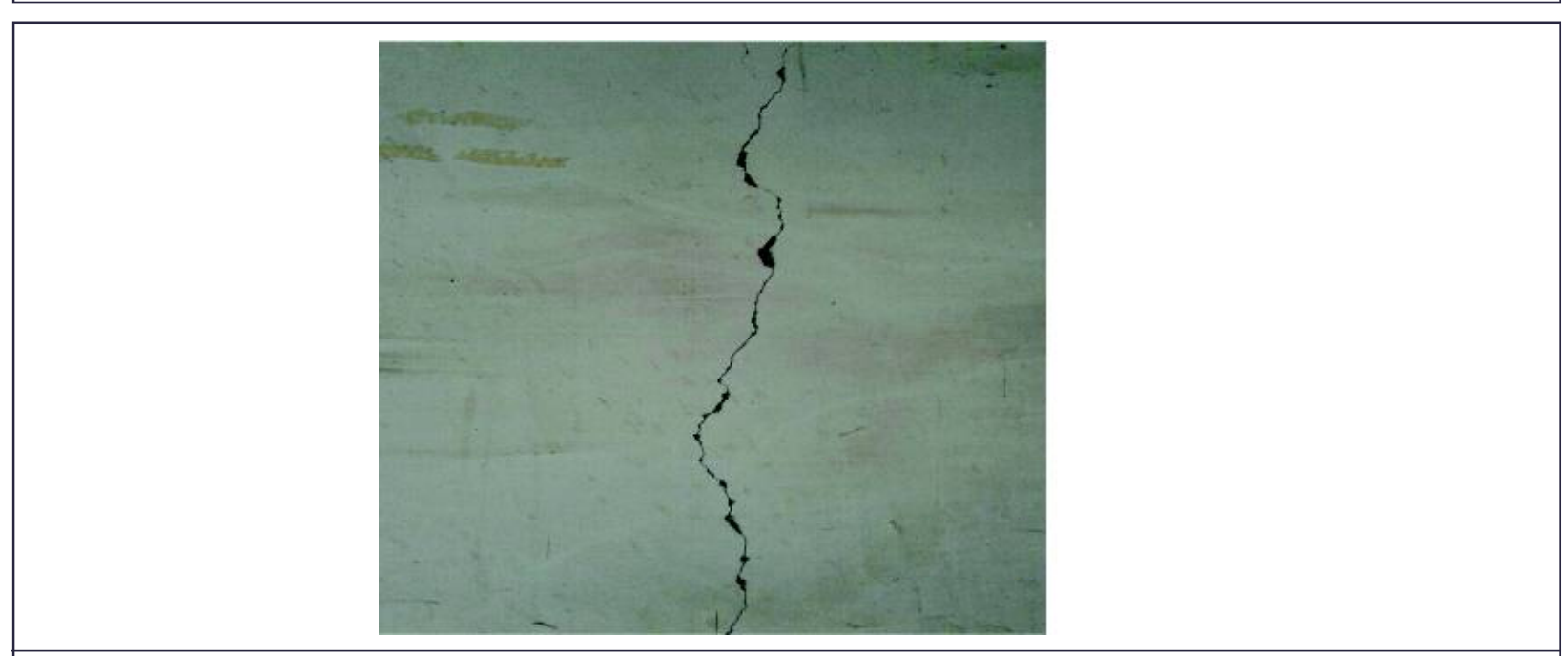

Figure 3: Cracks at juncture of slab and steel beam

Note: Width: above $2 \mathrm{~mm}$; Type: Non-structural, wide crack; and Causes: Due to joint, contraction occur between two walls. 


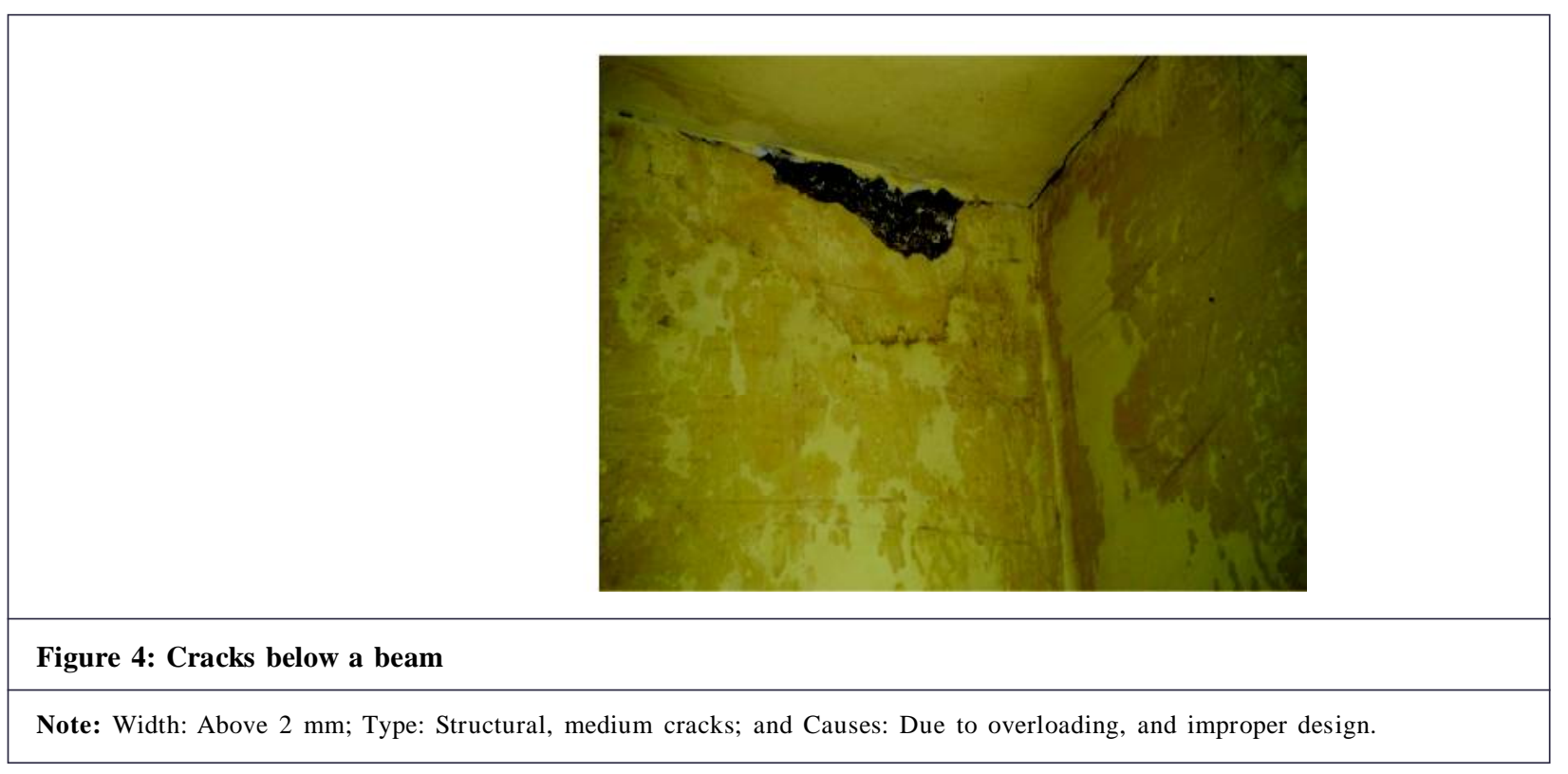

\section{Analysis of CCEM building, Raipur}

\subsection{Causes}

\subsubsection{Elastic deformation}

It occurs when a material strains under stress. When two materials (having different elastic properties) built together under the effect of load then different shear stresses in these materials create cracks at the junction. Dead and live loads are the main cause of elastic deformation in any structural components of a building. Preventive measures: Create slip joints under the support of concrete slab on walls. Provide horizontal movement joints between the top of brick panel and reinforced cement concrete beam/slab.

\subsubsection{Thermal movement}

Most materials expand when they are heated, and contract when they are cooled. The expansion and contraction with changes in temperature occur regardless of the structure's cross-sectional area. It is one of the most potent causes of cracking in buildings which need attention.

Preventive measures: Joints should be constructed like construction joints, expansion joints, control joints and slip joints. The joints should be planned at the time of design and be constructed carefully.

\subsubsection{Chemical reaction}

Chemical reactions may occur due to the materials used to make the concrete or materials that come into contact with the concrete after it has hardened. Concrete may crack with time as the result of slowly developing expansive reactions between aggregate containing active silica and alkalis derived from cement hydration, admixtures or external sources.

Preventive measures: If sulphate content in soil exceeds $0.2 \%$ or in ground water exceed 300 ppm, use very dense concrete and either increase richness of mix to $1: 1 / 5: 3$ and to prevent cracking due to corrosion in reinforcement it is desirable to specify concrete of richer mix for thin sections in exposed locations.

\subsubsection{Shrinkage}

Most of the building materials expand when they absorb moisture from atmosphere and shrink when they are dry. Shrinkage can be of plastic or dry. The factors causing shrinkage in cement concrete and cement mortar and their preventions are as following:

(1) Excessive water: The quantity of water used in the mortar mix can cause shrinkage. Vibrated concrete has less quantity of water and lesser shrinkage than manually compacted concrete.

Preventive measures: Use minimum quantity of water required for mixing cement concrete or cements mortar according to water cement ratio. Cement concrete is never allowed to work without mechanical mix and vibrator. 
(2) Quantity of cement: As a general rule, the richer the mix is, the greater the shrinkage/drying will be.

Preventive measures: Do not use excessive cement in the mortar mix.

\subsubsection{Foundation movement and settlement of soil}

Shear cracks in buildings occur when there is large differential settlement of foundation and it may be either due to the following reasons:

- Unequal bearing pressure under different parts of the structure.

- Bearing pressure on soil being in excess of safe bearing strength of the soil.

- Low factor of safety in the design of foundation.

- Local variation in the nature of supporting soil.

Preventative measure: The design of foundation should be based on sound engineering principles and good practice.

\subsubsection{Earthquake}

Crack may occur due to sudden shift in lower layer of the earth. The voids in the earth might have suddenly collapsed and be filled with soil from the above. Many geological events can trigger earth movements but is continuous movement.

Preventive measures: Construct the foundation of buildings on firm ground while doing construction. Tie up the building with connecting beams at foundation level, door level and roof level.

\subsubsection{Vegetation}

Fast growing trees in the area around the walls can sometimes cause cracks in walls due to expansive action of roots growing under the foundation. The cracks occur in clay soil due to moisture contained by roots.

Preventive measure: Do not grow trees too close to the building. Remove any saplings of trees as soon as possible if they start growing in or near of walls.

\subsubsection{Poor construction practices}

There is a general lack of good construction practices either due to ignorance, carelessness, greed or negligence. For a healthy building it is absolutely necessary for the construction agency and the owner to ensure good quality materials selection and good construction practices.

Preventive measure: Proper monitoring and use of good quality of materials is required at the time of construction.

\section{Methodology to prevent cracks}

\subsection{Epoxy injection}

Epoxy injection is an economical method of repairing non-moving cracks in concrete walls, slabs, columns and piers as it is capable of restoring the concrete to its pre-cracked strength. The technique generally consists of establishing entry and venting ports at close intervals along the cracks, sealing the crack on exposed surfaces, and injecting the epoxy under pressure.

\subsection{Routing and sealing}

In this method, the crack is made wider at the surface with a saw or grinder, and then the groove is filled with a flexible sealant. This is a common technique for crack treatment and it is relatively simple in comparison to the procedures and the training required for epoxy injection. It can be done on vertical surfaces and curved surface.

\subsection{Stitching}

This method is done to provide a permanent structural repairs solution for masonry repairs and cracked wall reinforcement. It is done by drilling holes on both sides of the crack, cleaning the holes and anchoring the legs of the staples in the holes with a non-shrink grout.

\subsection{Drilling and plugging}

This technique is only applicable when cracks run in reasonable straight lines and are accessible at one end. This method is mostly used to repair vertical cracks in retaining walls. 


\subsection{Gravity filling}

Low viscosity monomers and resins can be used to seal cracks with surface widths of 0.001 to 0.08 in by gravity filling. High molecular weight methacrylates, urethanes, and some low viscosity epoxies have been used successfully.

\subsection{Dry packing}

It is the hand placement of a low water content mortar followed by tamping or ramming of the mortar into place and also helps in producing intimate contact between the mortar and the existing concrete.

\subsection{Polymer impregnation}

Monomer systems can be used for effective repair of some cracks. A monomer system is a liquid consisting of monomers which will polymerize into a solid. The most common monomer used for this purpose is methyl methacrylate.

\section{Controlling measures}

\subsection{Measures for controlling cracks due to shrinkage}

- To avoid cracks in brickwork on account of initial expansion, a minimum period varying from 1 week to 2 weeks is recommended by authorities for storage of bricks after these are removed from Kilns.

- Shrinkage cracks in masonry could be minimized by avoiding use of rich cement mortar in masonry and by delaying plaster work till masonry has dried after proper curing and has undergone most of its initial shrinkage.

- Use of precast tiles in case of terrazzo flooring is an example of this measure. In case of in-situ/terrazzo flooring, cracks are controlled by laying the floor in small alternate panels or by introducing strips of glass, aluminum or some plastic material at close intervals in a grid pattern, so as to render the shrinkage cracks imperceptibly small.

- In case of structural concrete, shrinkage cracks are controlled by use of reinforcement, commonly termed as 'temperature reinforcement'. This reinforcement is intended to control shrinkage as well as temperature effect in concrete and is more effective if bars are small in diameter and are thus closely spaced, so that, only thin cracks which are less perceptible, occur.

- To minimize shrinkage cracks in rendering/plastering, mortar for plaster should not be richer than what is necessary from consideration of resistance to abrasion and durability

\subsection{Measures for controlling cracks due to thermal variations}

- Wherever feasible, provision should be made in the design and construction of structures for unrestrained movement of parts, by introducing movement joints of various types, namely, expansion joints, control joints and slip joints.

- Even when joints for movement are provided in various parts of a structure, some amount of restraint to movement due to bond, friction and shear is unavoidable. Concrete, being strong in compression, can stand expansion but, being weak in tension, it tends to develop cracks due to contraction and shrinkage, unless it is provided with adequate reinforcement for this purpose. . Members in question could thus develop cracks on account of contraction and shrinkage in the latter direction. It is, therefore, necessary to provide some reinforcement called "temperature reinforcement" in that direction (Chand, 2008).

- Over flat roof slabs, a layer of some insulating material or some other material having good heat insulation capacity, preferably along with a high reflectivity finish, should be provided so as to reduce heat load on the roof slab.

- In case of massive concrete structures, rise in temperature due to heat of hydration of cement should be controlled.

- Provision of joints in structure.

\subsection{Measures for prevention of cracks due to creep}

Though it may not be possible to eliminate cracking altogether, following measures will considerably help in minimization of cracks due to elastic strain, creep and shrinkage:

- Use concrete which has low shrinkage and low slump.

- Do not adopt a very fast pace of construction.

- Do not provide brickwork over a flexural RCC member (beam or slab) before removal of centering, and allow a time interval of at least two weeks between removal of centering and construction of partition or panel wall over it. 
- When brick masonry is to be laid abutting an RCC column, defer brickwork as much as possible.

- When RCC and brickwork occur in combination and are to be plastered over, allow sufficient time (at least one month) to RCC and-brickwork to undergo initial shrinkage and creep before taking up plaster work. Also, either provide a groove in the plaster at the junction or fix a $10 \mathrm{~cm}$ wide strip of metal mesh or lathing over the junction to act as reinforcement for the plaster (Central Building Research Institute, 1984).

- In case of RCC members which are liable to deflect appreciably under load, for example, cantilevered beams and slabs, removal of centering and imposition of load should be deferred as much as possible (at least one month) so that concrete attains-sufficient strength, before it bears the load.

\subsection{General measures for chemical attack}

- In case of structural concrete in foundation, if sulphate content in soil exceeds $0.2 \%$ or in ground water exceed $300 \mathrm{ppm}$, use very dense concrete and either increase richness of mix to 1:1/5:3 or use sulphate resisting portland cement/super-sulphated cement or adopt a combination of the two methods depending upon the sulphate content of the soil.

- For superstructure masonry, avoid use of bricks containing too much of soluble sulphates (more than $1 \%$ in exposed situations, such as parapets, free standing walls and masonry in contact with damp soil as in foundation and retaining walls; and more than 3\% in case of walls in less exposed locations) and if use of such bricks cannot be avoided, use rich cement mortar $(1: 1 / 2: 4.5$ or $1: 1 / 4: 3)$ for masonry as well as plaster or use special cements mentioned earlier and take all possible precautions to prevent dampness in masonry.

- To prevent cracking due to corrosion in reinforcement and premature deterioration, it is desirable to specify concrete of richer mix (say 1:1/5:3) for thin sections in exposed locations and to take special care about grading, slump, compaction and curing of concrete (Gambhir, 2005).

\section{Conclusion}

The potential causes of crack can be controlled if proper consideration is given to construction material and technique to be used. If we focus on the major causes to cracks in our building and take their preventive measures initially, we will able to minimize the problem of cracking in our structure.

Suggestions: There are so many structures which have failed due to occurrence of cracks and these failures caused a huge loss of life and property so if cracks get identified suitable remedial measures should be taken as soon as possible.

\section{Acknowledgment}

This research paper is made possible through the help and support from our teachers and friends. Especially, we are thankful to our project guide Prof. Bhumika Das (Head of Civil Department). We would also like to thank for her valuable guidance.

\section{References}

CBRI, Roorkee. (2011). Remedial measures of cracked buildings in expansive soil areas. http://krc.cbri.res.in:8080/dspace/ bitstream/123456789/253/1/BRN56.pdf

Central Building Research Institute, R. (1984). Handbook on causes and prevention of Cracks. IS. SP. 25.

Chand, S. (2008). Cracks in buildings and their remedial measures. Indian Concrete Journal.

Gambhir, M. L. (2005). Concrete technology (3rd ed.). New Delhi: The McGraw-Hill Companies.

Cite this article as: Bhumika Das, Kumar U mnag and Gopal M urthy (2021). Study on causes of cracks and its preventive measures in concretestructures of CCEM building, Rai pur. International J ournal of A rchitecture and Planning. 1 (1), 30-36. doi: 10.51483/ IJARP.1.1.2021.30-36. 